\title{
LA REPERCUSIÓn DE LAS AMPLIACIONES AL ESTE EN LA SEGURIDAD Y DEFENSA DE LA UE
}

\author{
The Impact of the European Eastern Enlargement \\ in the EU Security and Defence
}

\author{
Prof. Dra. Gracia Abad Quintanal \\ Profesora Agregada de Relaciones Internacionales. Universidad Nebrija \\ E-mail: gabad@nebrija.es
}

Si bien es innegable que la incorporación a la Unión Europea de algunos de los antiguos miembros del Pacto de Varsovia ha derivado también en una diversidad aún mayor de planteamientos y percepciones que hacen aún más difícil, si cabe, el progreso de la integración en un ámbito que se ha revelado como especialmente sensible desde un primer momento, el de la seguridad y la defensa, no es menos cierto que el nuevo contexto estratégico resultante del fin de la Guerra Fría supuso un impulso a la cooperación en este ámbito.

Con todo, es evidente que la ampliación de la organización ha condicionado algunos de los planteamientos de la UE respecto de determinadas cuestiones y de manera especial:

» La aproximación que debe adoptar y mantener la UE respecto de la Federación Rusa y sus políticas y movimientos estratégicos.

» La posición que debe adoptar la UE respecto de los conflictos (des)congelados en Europa: Kosovo, Georgia y Ucrania.

Con vistas a analizar estas cuestiones, este trabajo revisará la evolución de la cooperación en materia de seguridad y defensa en el seno de la UE desde el final de la Guerra Fría, analizará lo que la ampliación al este ha supuesto tanto para la Unión como para los nuevos miembros, especialmente en materia de seguridad y defensa, y profundizará en cómo dicha ampliación ha condicionado la implicación de la UE en algunos de los conflictos surgidos desde el fin de la Guerra Fría.

Seguridad; defensa; UE; ampliación al este; Guerra Fría.

Security; defense; EU; eastern enlargement; cold war.

Key mords

0 Autora

Resumen 
Although the EU membership of some former Warsaw Pact members certainly entailed a greater diversity of perceptions and approach which has made progress in the sensible realm of security and defence even more difficult, it is also true that the new strategic context resulting from the end of the Cold War meant an important stimulus in this realm.

All in all, it is clear that the enlargement has influenced EU approaches to certain issues, particularly:

» EU's approach toward the Russian Federation and its policies and strategic movements

"EU's approach towards the so called frozen conflicts in Europe: Kosovo, Georgia and Ukraine

In order to deal with this issue, in this paper we will analyse the evolution of Security and Cooperation in the EU after the Cold War; the implications of the Eastern Enlargement for the European Union as well as for the new members, particularly in security and defense matters; and the manner in which such enlargement has conditioned EU involvement in some of the conflicts which have arisen since the end of the Cold War.

\section{Introducción}

Si buscamos los orígenes del deseo europeo de avanzar en la integración en materia de seguridad y defensa debemos remontarnos no al final, sino al comienzo de la Guerra Fría. En efecto, no podemos olvidar la Comunidad Europea de Defensa o Plan Plevén (1952), el Plan Fouchet (1957) o la Cooperación Política Europea (1969) (Ahijado Quintillán, 2000, p. 191) que, si bien acabaron en fracaso (Mazziotti, 2014), fueron intentos serios de ir profundizando en la cooperación en la materia entre los Estados del continente.

Sin embargo, el fin de la Guerra Fría y el nuevo contexto estratégico que, como después recogerán las sucesivas estrategias de seguridad de la $U^{1}$, se iba a abrir con él iban a permitir un salto cualitativo en estos esfuerzos, impensable mientras el continente europeo se encontraba dividido (Smith, 2009, pp. 255-262). Ese cambio se pone de manifiesto de forma especialmente clara en 1992 con la incorporación de las misiones Petersberg en el marco de la Unión Europea Occidental (UEO) y la aprobación del Tratado de Maastricht.

Ahora bien, ese nuevo escenario, si bien iba a servir de acicate a los Estados europeos para avanzar en este terreno, también iba a generar nuevos obstáculos y desafíos derivados por una parte de la ampliación de la Unión Europea como consecuencia de la incorporación a la misma de buena parte de los antiguos Estados de Europa Central y Oriental y, por otra, de la emergencia de nuevos conflictos, frecuentemente en la periferia europea, y nuevos desafíos a la seguridad europea.

Y es que, como han planteado desde los años noventa diferentes teorías, no son pocas las consecuencias de la ampliación. Así, no han sido pocos los autores que han planteado potenciales efectos perversos de la misma para la profundización del proceso de integración, señalando que la ampliación dificultaría la dinámica de spill-over que impulsaba la integración, que existía el riesgo de que se debilitara el acervo comunitario, que se incrementaría la heterogeneidad o que

l Estrategia de Seguridad Europea, "Una Europa segura en un mundo mejor", Consejo de la Unión Europea, diciembre de 2003. También Estrategia Global Para la Política Exterior y de Seguridad de la Unión Europea, "Una visión común, una actuación conjunta: una Europa más fuerte”, Consejo de la Unión Europea, 2016. 
los procesos de toma de decisiones y las reformas se verían dificultados al tiempo que se tendería a una mayor complejidad administrativa (Wienner y Díez, 2004, p. 238).

Pues bien, entre todos esos posibles efectos, en relación con las cuestiones de seguridad y defensa y, por lo que respecta a los objetivos de este trabajo, dos son las principales consecuencias de la ampliación a tener en cuenta: su efecto en el spill-over y la creciente heterogeneidad en términos de gobernanza y manejo de lo político.

En lo que hace al spill-over, neoneofuncionalistas como Philippe C. Schmitter apuntaban que la ampliación se traduciría en el debilitamiento y retraso de tal dinámica (algo que, ya de entrada parece especialmente plausible en un ámbito tan sensible como el de la seguridad y defensa) a menos que las cuestiones que serían objeto de tal spill-over se introduzcan como parte de la negociación en el proceso de adhesión (Schmmitter, 2004, p. 70), cosa que no ocurrió.

Por lo que respecta a la creciente heterogeneidad, desde la perspectiva de la gobernanza se hizo ver que sería más difícil tanto la adopción de decisiones en el marco del Consejo como el establecimiento de normas estrictas y uniformes para todos los Estados, lo que haría necesario un grado mucho mayor de flexibilidad si se querían evitar bloqueos. Todo ello redundaría en el fortalecimiento de la autonomía de los miembros de la UE y el debilitamiento de la integración.

Por lo demás planteaban, al menos como posibilidad, la aparición de una fractura entre Estados europeístas y euroescépticos, que podría acabar por tener su reflejo incluso en el Parlamento Europeo de la mano de los partidos de la antigua Europa Central y oriental más opuestos a la integración (Jachtenfuchs y Kohler-Koch, 2004, p. 112).

A la luz de estas reflexiones de los distintos teóricos sobre las consecuencias potenciales de la ampliación y, a los efectos de este trabajo, cabe preguntarse cha dificultado la ampliación una dinámica de spill-over que supusiera un avance en la integración en la UE en materia de seguridad y defensa? ¿Se ha revelado más difícil la adopción de decisiones en este ámbito? ¿Se ha tendido en lo que tiene que ver con la seguridad y la defensa a un mayor nivel de flexibilidad? ¿Cabe hablar en el ámbito que nos ocupa de una fractura entre Estados miembros europeístas y euroescépticos?

Con vistas a responder a estas preguntas estas páginas tratan de analizar la evolución de la cooperación en materia de seguridad en el marco de la Unión Europea tratando de ver si se progresa o no hacia la integración en este ámbito; los procesos de toma de decisiones y las dificultades que han podido aparecer en ellos; y la existencia o no de posturas marcadamente distintas en favor y en contra de la europeización de las cuestiones de seguridad y defensa. Todo ello prestando atención, de manera especial, en relación con todas estas cuestiones al papel jugado por los nuevos Estados miembros de la UE que formaron parte con anterioridad del Pacto de Varsovia, en la medida en que dichos Estados parecen haber condicionado a la organización en dos aspectos:

» La aproximación que debe adoptar y mantener la UE respecto de la Federación Rusa y sus políticas y movimientos estratégicos.

» La posición que debe adoptar la UE respecto de los conflictos (des)congelados en Europa: Kosovo, Georgia y Ucrania.

Con ese fin, se hará en primer lugar un repaso de la evolución de la cooperación en materia de seguridad y defensa desde el fin de la Guerra Fría en el seno de la UE; en segundo lugar se analizarán las implicaciones de la ampliación de la UE hacia el Este tanto para dicha organización como para los nuevos Estados miembros y, por último, se profundizará en cómo la evolución de

\section{¿Cabe hablar en el ámbito que nos ocupa de una fractura entre Estados miembros europeístas y euroescépticos?}


la cooperación europea en materia de seguridad y defensa y, en especial, la participación en ella de los nuevos miembros han condicionado la implicación de la UE en algunos de los conflictos surgidos desde el fin de la Guerra Fría.

\section{De la PESC a la PCSD: la evolución de la cooperación en materia de seguridad y defensa en la Unión Europea}

Como decíamos más arriba, el fin de la Guerra Fría supuso un impulso considerable a las cuestiones de seguridad y defensa en el marco de la Unión Europea como consecuencia, en parte, del temor a una retirada de buena parte de los efectivos con que Estados Unidos contaba en Europa y, en parte, de las crecientes demandas para que los europeos tuvieran una participación cada vez más destacada en las operaciones de paz (Smith, 2004, p. 41). Una realidad que, si se piensa no es sino una constante en la profundización europea en materia de seguridad y defensa pues de modo recurrente —y la situación actual no es precisamente una excepciónson los temores europeos a un descenso del compromiso estadounidense los que sirven de impulso a los avances en la propia defensa europea.

Pues bien, una revisión de la evolución de la cooperación en materia de seguridad y defensa en la UE desde el final de la Guerra Fría nos permite identificar tres momentos clave que han permitido los tres grandes impulsos que dicha cooperación ha recibido hasta el momento:

\subsection{1-1992: El Tratado de Maastricht}

El cambio en el contexto internacional resultante del fin de la Guerra Fría, unido al salto hacia adelante que supone la creación de la Unión Europea, iba a generar el contexto ideal para los primeros años en que cabe considerar que se produce un avance sustantivo de la cooperación europea en materia de seguridad y defensa.

El primer paso en ese sentido es sin duda la creación de la Política Exterior y de Seguridad Común como uno de los tres pilares — el segundo- en que, en función del Tratado de Maastricht aprobado en 1992, se iba a basar la naciente UE (Mazziotti, 2014). Una política de seguridad que, no obstante, y como es comprensible dado el momento de que hablamos, iba a ser puramente intergubernamental y, por tanto, compatible con las políticas de seguridad de los Estados miembros.

Con todo, el Tratado de Maastricht también supuso un incremento de la influencia de la Comisión en la Política Exterior y de Seguridad Común, como demuestra la creación de una nueva Dirección General encargada específica de tales tareas (Sjursen, 1999).

Por otra parte, era relevante el hecho de que se permitiera a la UE contar con la Unión Europea Occidental para la ejecución de decisiones que tuvieran implicaciones en el ámbito de la defensa. De hecho, ese espíritu es el que permitió, de la mano de la declaración del mismo nombre ${ }^{2}$, la incorporación de las misiones Petersberg — tareas

\section{El Tratado de Maastricht también supuso un incremento de la influencia de la Comisión en la Política Exterior y de Segunidad Común}


humanitarias de rescate, de mantenimiento de la paz, de establecimiento de la paz y de gestión de crisis - a los "instrumentos" de la UE, aunque fuera en el marco de la UEO.

Tales avances iban a generar un clima de optimismo que llevaría al Ministro de Asuntos Exteriores de Luxemburgo, que ostentaba en junio de 1991 la presidencia rotatoria del Consejo de Ministros a decir que era "la hora de Europa... no la hora de los americanos" (Merlingen, 2012, p. 33).

Con todo, esos primeros pasos no iban a ser suficientes para acabar con la debilidad de la UE en materia de seguridad y defensa, como los conflictos en los Balcanes se encargarían de poner de manifiesto. En efecto, en ese contexto se hizo tristemente evidente que la Unión Europea era incapaz de hacer frente a situaciones de ese tipo toda vez que carecía de las capacidades necesarias para una acción verdaderamente seria en materia de prevención de conflictos, gestión de crisis o mantenimiento de la paz (Keukeleire y Macnaughtan, 2008, p. 16).

\subsection{7-1 998: El Tratado de Ámsterdam y la Declaración Franco-Británica de Saint-Malo}

Se iba a tratar de una fase decisiva, empezando por el propio tratado, pues por primera vez se iba a plantear el uso de medios militares para alcanzar las metas de la Unión Europea (Mazziotti, 2014).

Los cambios principales incorporados por el Tratado de Ámsterdam fueron:

» La introducción del Alto Representante para la Política Exterior y de Seguridad Común, llamado a dar a la PESC mayor continuidad y visibilidad frente a terceros Estados y a ayudar al Consejo de Ministros en su aplicación. El Alto Representante tenía además el cometido de dar apoyo a la Unidad de Planificación y Alerta Temprana, creada poco antes. El del establecimiento de la figura del Alto Representante es quizás el más importante de los cambios introducidos por este tratado.

» Las estrategias comunes, que tuvieron entre sus primeros "destinatarios" a Rusia (junio de 1999) y a Ucrania (diciembre de 1999), pero que desaparecerían pronto como consecuencia de sus escasos resultados (Bindi, 2012, p. 33).

» La facilitación del procedimiento de voto en el Consejo, dando más espacio a las decisiones por mayoría cualificada al menos en aquellos pasos en que ya hay un acuerdo previo para una acción o posición común.

» La capacidad con que en lo sucesivo iba a contar la Presidencia para negociar acuerdos internacionales relativos a la Política Exterior y de Seguridad Común (Pérez Bernardez, 2010, p. 270).

Frente a todo ello el Tratado de Ámsterdam sí supuso un cierto retroceso en lo que hace al papel de la Comisión, en buena medida como consecuencia de las reticencias de los Estados miembros, pero también fruto de la falta de legitimidad de que la Comisión podía adolecer al percibirse que no estaba sujeta a procedimiento democrático de rendición de cuentas alguno (Sjursen, 1999).

Ahora bien, el Tratado de Ámsterdam sí permitió una relación mucho más estrecha entre la Unión Europea y la Unión Europea Occidental con vistas a una posible integración de la UEO 
en la UE cuando el tratado de la primera se extinguiera. Una fusión que muchos hubieran deseado que se produjera ya entonces, pero que no fue posible por la oposición del Reino Unido (Smith, 2004, p. 45) que, con Margaret Thatcher como primera ministra y ya desde la invasión de Kuwait a manos de Irak en 1990, había evidenciado desacuerdos en materia de seguridad y defensa con otros miembros de las entonces Comunidades Europeas entre los que destacaba, precisamente, la cuestión de si la UEO debía fusionarse o no con la UE (Bindi, 2012, p. 24). Con todo, en lo que representaba un paso en esa dirección, las misiones Petersberg sí fueron incluidas en el Tratado de Ámsterdam.

Por lo demás se clarificaría por fin la cuestión presupuestaria y la PESC se financiaría con cargo al presupuesto de la CE, aunque dejando fuera las operaciones militares y de defensa.

Por otra parte, y en buena medida como consecuencia de la frustración derivada de la incapacidad puesta de manifiesto en el periodo anterior, especialmente en el contexto del conflicto de Kosovo, en esta fase la UE va a tratar de reforzar sus capacidades militares, especialmente en lo relativo a la gestión de crisis y el mantenimiento de la paz.

En efecto, la Declaración Franco-Británica de Saint-Malo o Declaración Conjunta sobre la Defensa Europea de diciembre de $1998^{3}$, posible por el cambio de actitud del Reino Unido derivado de la llegada al gobierno del laborista Tony Blair (Smith, 2004, p. 46), iba a suponer un elemento decisivo para dotar de operatividad al Tratado de Ámsterdam (Cornish y Edwards, 2001, p. 588) y dar el "pistoletazo de salida" en esta materia, pues en ella se señalaba que "la UE debe tener capacidad autónoma de acción, respaldada por fuerzas militares creíbles, medios para decidir usarlas y disposición de hacerlo” y, lo que es más importante, iba a contar con el respaldo de los Estados miembros, incluidos los neutrales, para su desarrollo (Smith, 2004, p. 46; Jones B., 2020, p. 7). Se trataba, en otras palabras y como señala Ozal Hopi de desarrollar elementos de hard power que reforzaran y dieran credibilidad al soft power de la UE (Hopi, 2011, 2012, p. 9).

Unos cambios que, para muchos, iban a significar el abandono por parte de la Unión Europea de su carácter de potencia civil (Keukeleire y Macnaughtan, 2008, p. 16), resultante en buena medida, como señala Francois Duchêne, del momento de estancamiento que supone la guerra fría (Duchêne, 1973, p. 32), y que dejarían a muy pocos indiferentes, pues si desde cierta perspectiva suponía el abandono por parte de la UE de su naturaleza pacífica, los atlantistas veían lo con temor en la medida en que pudiera poner en cuestión la primacía de la OTAN en materia de defensa europea y los más nacionalistas no acababan de aceptar la idea de compartir activos militares en el marco de la UE (Mazziotti, 2014).

En ese sentido, en el Consejo Europeo de Colonia de junio de $1999^{4}$ se fijó la meta de establecer una Política Europea de Seguridad y Defensa insistiendo en algunas de las cuestiones clave que ya había planteado la Declaración de Saint-Malo. Al propio tiempo anunció la desaparición de la UEO a comienzos de 2001 y la inclusión de sus estructuras y personal en la UE (Mazziotti, 2014).

3 Declaración franco-británica de St. Malò, Declaración Conjunta sobre la Defensa Europea, 4 de diciembre de 1998, recuperado de internet. https:/www.cvce.eu/content/publication/2008/3/31/f3cdl6fb-fc37-4d52-936fc8e9bc80f24f/publishable_en.pdf

4 Declaración del Consejo Europeo sobre el refuerzo de la Política Europea de Seguridad y Defensa, Consejo Europeo de colonia, Conclusiones de la Presidencia, 3 y 4 de junio de 1999, recuperado de internet. http://www.europarl. europa.eu/summits/kol2_es.htm\#bilIII

\section{Elementos de hard pomer que reforzaran y dieran credibilidad al soft pomer de la UE}


Junto a todo ello el Consejo Europeo de Colonia acordó la redefinición del Eurocuerpo, compuesto por Francia, Alemania, Bélgica, Luxemburgo y España para transformarlo en una fuerza de reacción rápida (Sjursen, 1999).

Unos meses después, el Consejo Europeo de Helsinki ${ }^{5}$ supondría nuevos avances, esta vez con implicaciones incluso en términos operativos (Cornish y Edwards, 2001, p. 588). Así, si por una parte se insistió en la importancia de que la UE contara con "capacidad de decisión autónoma y, cuando la OTAN en su conjunto no estuviera implicada, lanzar y liderar operaciones militares en respuesta a crisis internacionales", por otra, quedo fijado el llamado "Helsinki Headline Goal" en virtud del cual los Estados miembros que, voluntariamente, desearan participar en operaciones lideradas por la UE se comprometían a ser capaces para 2003 de desplegar en 60 días hasta 60.000 efectivos sostenibles sobre el terreno durante un año y con capacidad para desarrollar todo el abanico de misiones Petersberg. Junto a ello acordaron la creación de un Comité Político y de Seguridad, un Comité Militar y un Estado Mayor en el marco del Consejo.

En definitiva, cabe pensar que iba surgiendo ya una cierta "cultura estratégica europea” (Cornish y Edwards, 2001, p. 587) y que se estaban dando pasos que permitieran avanzar desde una Política Exterior y de Seguridad Común que contenía muchos elementos principalmente retóricos a una Política Europea de Seguridad y Defensa mucho más orientada a realizaciones prácticas (Keukeleire y Macnaughtan, 2008, p. 57), pero en el marco de la cual unas verdaderas fuerzas armadas europeas continuarían siendo las grandes ausentes. Dicha PESD sería finalmente declarada operativa en diciembre de 2001, con ocasión de la Declaración de Laeken

Tales pasos no se detendrían y, de hecho, se seguiría avanzando en esa línea como muestra la aprobación del Headline Goal 2010 por el Consejo Europeo en junio de 2004, que debía mucho a la experiencia derivada de la creciente amenaza terrorista y de los conflictos de Irak y Afganistán. En este sentido, se trataba de ser capaces de desplegar fuerzas en un plazo no superior a diez días desde la decisión de lanzar una operación. Dichas fuerzas, más allá de las misiones Petersberg, tendrían capacidad para participar en operaciones conjuntas de desarme y para apoyar a terceros Estados en la lucha contra el terrorismo y la reforma del ámbito de la seguridad.

Prueba del impulso del momento es además la voluntad de crear una Célula Civil-Military, una Agencia Europea de Defensa y de desarrollar una capacidad eficiente de transporte estratégico. Asimismo, se esperaba contar con Battle Groups (1500 efectivos desplegables en 15 días y de alta eficiencia) operativos para 2007 y un portaviones que estaría funcionando en 2008.

Unos avances que, sin embargo, iban a quedar en cierto modo en punto muerto como resultado del contexto de división que, especialmente en materia de seguridad y defensa iba a vivir la UE en los años siguientes y que no eran sino la consecuencia de la existencia de posiciones marcadamente divergentes entre los Estados miembros en relación con el conflicto de Iraq y, aunque en menor medida, también con el de Afganistán, ya mencionados. Unos conflictos que, por otra parte, también volvieron a poner de manifiesto las limitaciones de las capacidades militares de la Unión Europea.

5 Conclusiones de la Presidencia, Consejo Europeo de Helsinki 10 y 11 de diciembre de 1999, recuperado de internet. http://www.europarl.europa.eu/summits/hell_es.htm

6 Conclusiones de la presidencia, Consejo Europeo de Helsinki, 10 y 11 de diciembre de 1999, recuperado de internet. http://www.europarl.europa.eu/summits/hell_es.htm

7 Concusiones de la Presidencia, Consejo Europeo de Laeken, 15 y 16 de diciembre de 2001, recuperado de internet. https://www.consilium.europa.eu/media/20950/68827.pdf
Unos meses

después, el

Consejo Europeo

de Helsinki

supondría nuevos

avances, esta vez

con implicaciones incluso en términos operativos 


\section{2005-2017: El Tratado Constitucional y el Tratado de Lisboa}

Sin embargo, la UE acabaría retomando sus esfuerzos en el ámbito de la cooperación en materia de seguridad y defensa y prueba de ello es que el Tratado Constitucional ampliaba las misiones originales de Petersberg e introducía elementos de flexibilización de la PESD, una cláusula de solidaridad y nuevos elementos institucionales entre los que cabe destacar la Agencia Europea de Defensa.

Pese al fracaso del Tratado Constitucional, como consecuencia de los rechazos en referéndum en Francia y Países Bajos, tanto la ampliación de las misiones Petersberg como la creación de la Agencia Europea de Defensa se llevaron a efecto.

Por lo demás, fracasada la vía constitucional, los Estados miembros iban a volver al método tradicional y apostar por un nuevo tratado de reforma que acabaría por firmarse en Lisboa en diciembre de 2007, que mantenía mucho de lo previsto en el tratado constitucional y que incorporaría algunos de los mayores avances registrados hasta el momento en el ámbito de la seguridad y la defensa (Kocamaz, 2011, p. 470).

De hecho, prueba de la importancia que el nuevo tratado concedía a las cuestiones de política exterior y de seguridad es que las cuestiones relativas a la PESC fueron incluidas en el Tratado de la Unión Europea (Título V, artículos 23 a 46, disposiciones específicas de la PESC), mientras que las relacionadas con las restantes políticas quedaron recogidas en el Tratado de Funcionamiento de la UE. Una inclusión en el TUE, separada del resto de la acción exterior de la Unión (salvo lo que aparece en los artículos 21 y 22 del TUE) que perpetúa, pese a la desaparición de la estructura de pilares (Garrido, 2019, p. 426), la división entre la PESC (incluida la PCSD) y el resto de las relaciones exteriores (Pérez Bernardez, 2010, p. 268), algo que, como recuerda Carmela Pérez Bernárdez, reconoce el propio TUE al señalar en su artículo 24 que la PESC “se regirá por reglas y procedimientos especiales” (Pérez Bernardez, 2010, p. 273).

Se introducía así, en todo caso, por primera vez una sección específica en el tratado dedicada a las cuestiones de seguridad y defensa (Merlingen, 2012, p. 37) que iba a recibir una nueva denominación, Política Común de Seguridad y Defensa, haciendo hincapié precisamente en la importancia de poner en "común” un ámbito tan sensible como el que nos ocupa.

En ella se creaba la figura del Alto Representante de la Unión para Asuntos Exteriores y Política de Seguridad que unía a las funciones que habían caracterizado al Alto Representante para la PESC, las de vicepresidente de la Comisión encargado de la Política Exterior. Quedaban así por primera vez vinculadas aquellas materias de política exterior y de seguridad en que era competente el Consejo y eran eminentemente intergubernamentales con las que tenían un carácter más supranacional y eran responsabilidad de la comisión.

Junto a ello y, quizás más importante, el nuevo tratado ampliaba los ámbitos en los que era posible el establecimiento de "cooperaciones reforzadas", que ahora pasaban a incluir cuestiones de seguridad y defensa que habían quedado expresamente fuera con ocasión del Tratado de Niza, una vez más por deseo británico (Bindi, 2012, p. 34).

En esa misma línea, se preveía la creación de una "Cooperación Estructurada Permanente" llamada con el tiempo a hacer posible la "Autonomía Estratégica” (Saulnier, Anghel, Lazarou, y Wilson, 2020) y que, a diferencia de la cooperación reforzada, no iba a estar abierta a la participación de todos los Estados, sino solamente de aquellos que cumplieran con ciertos requisitos
Quedaban así por primera vez vinculadas aquellas materias de política exterior y de seguridad en que era competente el Consejo y eran eminentemente intergubemamentales con las que tenían un carácter más supranacional y eran responsabilidad de la comisión 
en términos de capacidades militares. Aquellos Estados que finalmente pudieran participar en dicha cooperación estructurada podrían profundizar en la colaboración en materia de seguridad y defensa incluso en términos de efectivos y capacidades militares.

Por otra parte, es tanto o más relevante la introducción de una cláusula de defensa colectiva (Arteaga, 2016, p. 5), en buena medida heredada de la existente en el artículo 5 del Tratado de la UEO, en virtud de la cual los Estados se obligan a ayudar a aquel que pudiera ser víctima de una agresión armada (Mangas Martín, 1989, p. 76), aunque con la salvedad de que ello no necesariamente altera la política de los Estados neutrales ni tampoco pretende entrar en contradicción con los compromisos de los Estados que son al mismo tiempo miembros de la UE y de la OTAN.

Junto a ella hay que mencionar la "Cláusula de Solidaridad" que permite que un estado miembro víctima de una catástrofe natural o un ataque terrorista reciba asistencia de los restantes Estados miembros o de la Unión en su conjunto.

A todas estas cuestiones hay que sumar una ampliación considerable del ámbito en el que cabía hacer uso de las misiones Petersberg que, no obstante, no ha evitado que la actuación de la UE en gestión de crisis se quedara muy por debajo de lo esperado (Garrido, 2019, p. 408).

Sin embargo, no podemos perder de vista que, desde esta última fase que acabamos de analizar y en lo sucesivo, los avances y la profundización de la cooperación en las siempre difíciles cuestiones de seguridad y defensa tendrían un condicionamiento más: la incorporación de los anteriormente llamados PECOS (Países de Europa Central y Oriental).

\section{La ampliación al este y el papel de los nuevos Estados miembros en materia de seguridad y defensa}

Desaparecida la Guerra Fría estaba claro que la UE tenía que ser parte en la trasformación del orden existente en los llamados Estados de Europa Central y Oriental. En su caso, la implicación pasaría en buena medida por abrir la puerta a la ampliación al este de la organización, dando pasos hacia la incorporación a la misma de algunos de esos Estados. Así, incluso los más escépticos con una posible ampliación acabaron por ceder ante quienes les recordaban que la Unión Europea no había sido nunca un club cerrado, que como había señalado el propio Parlamento Europeo el 4 de octubre de 2000 "la unificación de Europa en una zona de paz, seguridad, prosperidad y estabilidad tras su división a raíz de la ocupación soviética de Europa del Este sigue siendo la misión histórica y el objetivo último de sus políticas" (Pérez Sánchez, 2007, p. 214) y que no abrir las puertas a los entonces llamados PECOS (Estados de Europa Central y Oriental) sería difícil de justificar toda vez que entraba en contradicción con los principios fundamentales del propio proceso de construcción europea (Schimmelfennig, 2004). Por su parte, los PECOS pronto llegarían a la conclusión de que la integración en las organizaciones regionales y globales de carácter internacional era para ellos el mejor escenario de futuro (Smith, 2014, p. 256) y, en el caso de la UE, el que les permitiría compartir la prosperidad y centralidad de Europa Occidental (Gati, 2007, p. 113).

De hecho, al menos en el caso de la UE, la expectativa de integración en la organización se convertiría en el mejor acicate para que dichos Estados emprendieran reformas clave de carác-
Desaparecida la Guerra Fría estaba claro que la UE tenía que ser parte en la trasformación del orden existente en los llamados Estados de Europa Central y Oriental 
ter tanto económico como político (Smith, 2014, p. 64) que les permitieran cumplir con los criterios fijados en junio de 1993 con ocasión del Consejo Europeo de Copenhague ${ }^{8}$ con la idea de que la importante ampliación que se iba a acometer no supusiera un debilitamiento de la UE (Smith, 2014, p. 260). Dichos criterios serían sistema democrático, Estado de derecho, respeto a los derechos humanos y protección de las minorías, economía de mercado y capacidad para asumir las obligaciones derivadas de su condición de miembros. Unos criterios a los que se sumó uno más en 1995, de la mano del Consejo Europeo de Madrid ${ }^{9}$, en virtud del cual los socios debían aplicar el acervo comunitario y adaptarse al mismo.

Si bien tales criterios pueden parecer lógicos y, de hecho "simplemente" suponían la adaptación de los candidatos al funcionamiento del resto de los ya Estados miembros, no resultaban demasiado fáciles de cumplir para unos Estados deseosos de su "retorno a Europa” (Pérez Sánchez, 2007, p. 207) —no en vano ese retorno había significado durante décadas sacudirse el yugo de la dominación soviética y volver a establecer vínculos con el Occidente democrático (Wallace, 2000, p. 478) — que los subestimaron (Wallace, 2017, p. 81) y acababan de dejar atrás décadas de sistemas no democráticos y de economía planificada, lo que les llevó a pensar en ocasiones, no sin razón, que los entonces Estados miembros de la UE no eran demasiado partidarios de su incorporación e, incluso, que no se les estaba aplicando a ellos el mismo rasero que se había aplicado en el momento de su adhesión a los que habían accedido en anteriores ampliaciones al estatuto de miembros.

Por otra parte, no podemos olvidar que el proceso de integración supone una aproximación y concertación de políticas entre los Estados miembros, implicando incluso en algunos casos la cesión de soberanía (Pérez Bernárdez, 2010, p. 266). Si bien, en el caso concreto de las cuestiones de seguridad y defensa que nos ocupan aquí está última cuestión aún no está presente, esa dinámica de concertación, en su conjunto, bien puede resultar ardua para Estados que acaban de poner fin a décadas de control externo de sus políticas, incluidas —o, de modo especial—, las que tienen que ver con la proyección exterior, la seguridad y la defensa.

Así, esta realidad unida al hecho de que la necesidad de cumplir con los criterios fue el argumento para diferentes reformas, algunas no especialmente populares en los Estados miembros, inevitablemente sembraron la semilla del euroescepticismo en los nuevos Estados miembros (Smith, 2014, p. 260; Kolankiewicz, 1994, p. 479).

Con todo, tanto en lo que respecta a las élites como en relación con la población, la actitud respecto de la incorporación a la UE y el conjunto del proceso de integración europea en sí variaba notablemente de Estado a Estado, situándose quizá parte de las élites polacas y checas entre los más escépticos. Esa situación no solo se ha mantenido en el tiempo, como pone de manifiesto la tardanza de checos y polacos de cara a la ratificación del Tratado de Lisboa, sino que se ha ido agravando de la mano de la aparición de una nueva generación de líderes en los Estados de la antigua Europea Central y Oriental que nada tienen que ver con los que lideraron las transiciones. De hecho, en algunos casos, como el de Polonia, nos encontramos altos cargos, como Antoni Macierewicz, amigo personal de los hermanos Kaczynski y encargado del

8 Criterios de Adhesión o Criterios de Copenhague, recuperado de internet. https:/eur-lex.europa.eu/summary/ glossary/accession_criteria_copenhague.html?locale=es

9 Conclusiones de la Presidencia, Consejo Europeo de Madrid, 15 y 16 de diciembre de 1995, recuperado de internet. http://www.europarl.europa.eu/summits/mad3_es.htm no podemos olvidar que el proceso de integración supone una aproximación y concertación de políticas entre los Estados miembros, implicando incluso en algunos casos la cesión de soberanía 
desmantelamiento del servicio de inteligencia militar polaco que, ya en los noventa, mostraban abiertamente su oposición a la participación en la UE (Gati, 2009, pp. 109-111).

En este sentido, además, parecía claro desde un primer momento que en la medida en que los costes - económicos para empezar - de la participación en el proceso no fueran acompañados de beneficios evidentes o superaran con mucho a estos últimos, el sentimiento antieuropeo, acompañado de elementos de xenofobia y nacionalismo, podría ganar terreno y generarse un contexto mucho menos propicio (Smith, 2014, pp. 481-482), una situación que lamentablemente parece manifestarse en algunos casos.

Así, una y otra circunstancia demuestran que los teóricos de la gobernanza no se equivocaban al apuntar que la creciente heterogeneidad derivada de la ampliación podía traducirse en la aparición de una fractura — que es una realidad - entre europeístas y euroescépticos.

En otro orden de cosas, cabe mencionar también que la expectativa de la ampliación subyace también en buena medida a la aprobación en 2000 del Tratado de Niza y a las modificaciones que el mismo recogía, pues también la UE y su aparato institucional tenían necesidad de adaptarse a la nueva realidad derivada de una ampliación sin precedentes (Smith, 2014, pp. 256257), que suponía acoger nada menos que a trece nuevos Estados (Bindi, 2012, p. 34). En este sentido, el nuevo tratado recogía cuestiones tales como la ampliación de los ámbitos de la PESC susceptibles de ser decididos por mayoría cualificada o el refuerzo del papel de Comité Político y de Seguridad en operaciones de gestión de crisis.

En cualquier caso, quizás lo más interesante de cara a las cuestiones que nos ocupan en estas líneas es que esa apertura a la ampliación de la UE a los Estados de Europa Central y Oriental, en términos de seguridad y defensa suponía que pasaban a ser integrantes de la organización Estados que habían formado parte del Pacto de Varsovia (Keukeleire y Macnaughtan, 2008, p. 14), desaparecido en 1991 e, incluso, alguno que había sido parte integrante de la también extinta Unión Soviética.

En ese sentido, hay que decir que la ampliación no se produjo de modo repentino, sino que algunos pasos previos fueron permitiendo la adaptación de los nuevos Estados. Así, en 1994 a los antiguos Estados del Pacto de Varsovia se les ofrece el status de Socios Asociados en la Unión Europea Occidental, lo que hacía posible su participación en las misiones Petersberg, pero les dejaba fuera del marco de defensa colectiva que representaba la UEO (Bindi, 2012, p. 27). Asimismo, y en función de lo decidido en el Consejo Europeo de Essen en 1994 en el marco de la Estrategia de Preadhesión, los socios tomarían parte en un diálogo reforzado con la UE sobre cuestiones de Política Exterior y de Seguridad Común (Bindi, 2012, p. 29).

A partir de ahí y de acuerdo con la decisión adoptada en el Consejo Europeo de diciembre de 1997, el 31 de marzo de 1998 se iniciaron negociaciones para la adhesión con la República Checa, Estonia, Hungría, Polonia, Eslovenia y Chipre y un año después con Letonia, Lituania, Malta, Eslovaquia, Bulgaria y Rumanía, un impulso que se mantendría claramente con ocasión del Consejo Europeo de Helsinki.

La ampliación que, para decepción de Polonia, la República Checa y Hungría que pensaban que su camino hacia el ingreso en la UE sería mucho más breve y, desde luego más rápido que el de otros candidatos (Wallace, 2000, p. 476), se concretaría en 2004 con el ingreso de diez nuevos Estados de los cuales ocho pertenecían a Europa Central y Oriental, —República Checa, Estonia, Hungría, Polonia, Eslovenia, Chipre, Letonia, Lituania, Malta y Eslo- 
vaquia - y a los que seguirían Rumanía y Bulgaria en 2007, iba a hacer cambios necesarios en la conceptualización en materia de seguridad y defensa de la UE y también en términos institucionales.

Y es que esa ampliación implicaba el ingreso en la UE de nuevas sensibilidades en relación con algunas de las cuestiones que habían marcado la evolución de la Unión en este ámbito o un cambio en los equilibrios de fuerzas existentes entre los Estados miembros partidarios de determinados planteamientos y los que preferían otras opciones.

En este sentido, los nuevos Estados, en la dicotomía entre atlantistas y europeístas que había marcado - y sigue marcando- desde siempre la evolución de la cooperación en materia de seguridad y defensa, iban a inclinar claramente la balanza del lado de los atlantistas. Ya entonces, y esta es una dinámica que no iba sino a acrecentarse con el paso del tiempo, los nuevos Estados miembros desconfiaban de las capacidades militares y de disuasión de la UE mientras veían en la OTAN una garantía mucho mayor (Muti, 2018, p. 1) de su seguridad en particular frente a un vecino, Rusia, cuya conducta e intenciones futuras veían con recelo (Keukeleire y Macnaughtan, 2008, p. 61). Así, la aparición, como decíamos más arriba, de una fractura europeístas-euroescépticos estaba servida.

Buen ejemplo de todo ello es el caso de Polonia, un Estado europeo con un gasto militar considerable y con un territorio y una población razonablemente amplios que sin embargo ha estado poco implicado en los esfuerzos de la UE en materia de seguridad y defensa y que, de hecho, hasta hace poco tan solo se había vinculado a dos de los proyectos a desarrollar en el marco de la Cooperación Estructurada Permanente (Muti, 2018, p. 2).

Una circunstancia que pone de manifiesto que se ha materializado otro de los efectos no deseados de la mayor heterogeneidad derivada de la ampliación y sobre la que advertían los teóricos de la gobernanza: el refuerzo de la autonomía de los miembros y la aparición de dinámicas de mayor flexibilidad (como las mencionadas en el marco de la PESCO) para evitar bloqueos.

Por otra parte, el proceso no tiene por qué estar acabado si tenemos en cuenta que en junio de 2000, en el marco del Consejo Europeo de Feira ${ }^{10}$ y sobre la base del Pacto de Estabilidad para el Sudeste Europeo lanzado un año antes, se consideraría a los Estados de los Balcanes como potenciales candidatos a la adhesión, con lo que dejaba de ser impensable que los Estados resultantes de la desintegración de Yugoslavia, muchos de ellos entonces —e incluso ahoracaracterizados no solo por la debilidad sino por el conflicto, acabaran por ser un día miembros de la Unión Europea (Wallace, 2000, p. 477).

Una circunstancia que, siendo indudablemente positiva en relación con el mencionado "retorno a Europa" tiene potencialmente consecuencias negativas para el devenir del proceso de integración, como ya estamos viendo.

En otras palabras y, de acuerdo con los planteamientos neofuncionalistas, si el spill-over siempre fue esquivo en lo que a las cuestiones de seguridad y defensa se refiere, en este escenario de ampliación (inacabada aún) parece haberse vuelto casi una quimera.

10 Conclusiones de la Presidencia, Consejo Europeo de Santa María de Feira, 19 y 20 de junio de 2000, recuperado de internet. http://www.europarl.europa.eu/summits/fei2_es.htm
Si el spill-over siempre fue esquivo en lo que a las cuestiones de seguridady defensa se refiere, en este escenario de ampliación (inacabada aún) parece haberse vuelto casi una quimera 


\section{La nueva conflictividad tras el fin de la Guerra Fría: el papel de la Unión Europea}

Por otra parte, para tomar conciencia de la complejidad de la nueva situación, debemos tener presente, además, que el final de la Guerra Fría supone la aparición —y en algunos casos el resurgimiento - de un buen número de conflictos, notablemente diferentes, además, de los conflictos que habían caracterizado al periodo de Guerra Fría. Frente a aquellos se trata ahora, en la mayoría de los casos, de conflictos internos o, a lo sumo, regionales donde se entremezclan las causas de carácter étnico, religioso, político, etc., y donde los actores ya no son solo ejércitos regulares, sino paramilitares, milicias, grupos terroristas y criminales, etc. que compiten por la conquista del poder, por el dominio de un territorio, por el reconocimiento de su independencia, por el acceso a determinados recursos económicos, etc. (Keukeleire y Macnaughtan, 2008, p. 61; Kaldor, 1999).

Se trata, por otra parte, de conflictos sustancialmente más complejos que los anteriores para cuya gestión será necesario poner en juego algo más que fuerza militar, tal como los casos de Bosnia, Kosovo, Afganistán o Irak o Georgia, por mencionar algunos, han puesto de manifiesto (Keukeleire y Macnaughtan, 2008, p. 61), pues requieren que se generen estructuras políticas, económicas y sociales que permitan tratar de asegurar una paz duradera, y en los que la participación de terceros que contribuyan a la solución de dichos conflictos será cada vez más importante (Kocamaz, 2011, pp. 467-468).

Por otra parte, como consecuencia en parte de la propia ampliación (Cornish y Edwards, 2001, p. 598), algunos de esos conflictos van a tener lugar, además, en la periferia de la Unión Europea (Mazziotti, 2014) para la que se hará evidente, como para otros actores, la necesidad de estabilizar toda esa área si no quiere que la inestabilidad termine por afectarle negativamente a ella (Keukeleire y Macnaughtan, 2008, p. 15). De hecho, la propia Estrategia Europea de Seguridad "Una Europa Segura en un Mundo Mejor" aprobada en diciembre de $2003^{11}$, mencionada más arriba, recogía como una de las líneas prioritarias para la UE la gestión de su vecindario.

Será esa la necesidad que llevará a la Unión Europea a la creación de la Política de Vecindad, transformada en Asociación Oriental (Rodríguez Prieto, 2018) desde 2009, y orientada precisamente a la promoción de la estabilidad en su periferia.

En este sentido, por ejemplo, la Unión Europea se iba a convertir en un actor relevante cuando a finales de 2004 se produce la revolución en Ucrania, un Estado que, curiosamente, ya en 1991 fijaba entre sus dos prioridades estratégicas la incorporación a la UE (Wallace, 2017, p. 81). Del mismo modo, se vería obligada a nombrar Representantes Especiales de la UE en el Cáucaso Sur, Asia Central o Moldavia (Keukeleire y Macnaughtan, 2008, p. 61) por mencionar algunos casos de zonas inestables.

Ahora bien, esa aproximación proactiva de la Unión Europea, resultante más de la necesidad que del deseo de presencia europeo, va a llevar a una creciente tensión con otro actor clave en el actual contexto de seguridad europeo: Rusia.

En efecto, si tras el fin de la Guerra Fría, durante la década de los noventa, en buena medida por la influencia de los Estados de Europa Oriental que hicieron ver a la UE que la retórica de

11 Estrategia de Seguridad Europea, "Una Europa segura en un mundo mejor", Consejo de la Unión Europea, diciembre de 2003.

\section{La Unión Europea se iba a convertir en un actor relevante cuando a finales de 2004 se produce la revolución en} Ucrania, un Estado que, curiosamente, ya en 1991 fijaba entre sus dos prioridades estratégicas la incorporación a la UE 
Guerra Fría en absoluto podía resultar beneficiosa, las relaciones fueron relativamente cordiales (Ischinger, 2009) de la mano de iniciativas como las reuniones bilaterales dos veces al año o la adopción de una estrategia hacia Rusia, aprobada por el Consejo Europeo de Colonia de 1999, —documento donde, de hecho, se enfatizaba la importancia de esa cooperación para la seguridad regional y global; la posibilidad de que Rusia participara en misiones de la UEO y la importancia de la colaboración entre ambos actores en la construcción de una nueva arquitectura de seguridad europea en el marco de la OSCE- (Ruiz González, 2010, p. 230), con posterioridad la negativa de Rusia a lo que consideraba ser un socio junior; la propia ampliación de la UE percibida desde Rusia como un empeño de la Unión (Wallace, 2017, p. 81), cuando había sido más el resultado de la insistencia de los nuevos Estados miembros a pesar de las reticencias de los miembros occidentales de la UE y, desde luego, la política de vecindad de la UE que percibe como un desafío y la creciente asertividad de ambos, han resultado en tensiones cada vez mayores (Merlingen, 2012, p. 215).

Unas tensiones que no son sino la consecuencia del choque entre la Asociación Oriental de la UE (Rodríguez Prieto, 2018), centrada ahora en Georgia, Armenia, Azerbaiyán, Moldavia, Bielorrusia y Ucrania, —nuevos vecinos de la UE tras la última ampliación (Park, 2014) - y que, como decíamos más arriba no es sino el fruto de la necesidad de estabilizar su vecindario para evitar que la inestabilidad le acabe afectando a ella y la concepción rusa, en virtud de la cual el "extranjero próximo" es su área de influencia y en ella tiene cierto derecho de supervisión del curso de los acontecimientos (Merlingen, 2012, p. 217).

En este sentido, se hará pronto evidente que la aproximación de Rusia a los conflictos de esta zona está siempre orientada al mantenimiento de su influencia en la región sin que, a diferencia de la UE, tenga particular interés en la estabilidad de la misma.

Un problema añadido al que debe hacer frente la UE en el contexto de esas tensiones crecientes con la Federación Rusa es la falta de acuerdo acerca de cuál es la aproximación adecuada en este asunto existente entre los Estados miembros, algo tristemente frecuente en el seno de la Unión, por otra parte.

Con todo, la implicación de la UE en su vecindario y sus intentos de limitar la conflictividad en él, lejos de desaparecer, se han mantenido e, incluso se han incrementado con el tiempo. En este sentido, cabe mencionar el nombramiento de Representantes Especiales de la UE para Moldavia en 2005, para el Cáucaso Sur en 2003 y tras la Crisis de Georgia en septiembre de 2008. Del mismo modo, también desplegó una misión de restablecimiento del estado de derecho (EUJUST Themis) en Georgia durante el periodo 2004-2005, un equipo de apoyo en fronteras en 2005 y participó en la verificación del alto el fuego entre Rusia y Georgia en Octubre de 2008 (Merlingen, 2012, p. 224).

Así, la implicación de la UE en la región se ha mantenido firme incluso cuando abiertamente contradecía las preferencias de Rusia, como ocurrió con la oposición al plan de paz ruso en respuesta al problema separatista en Moldavia en 2003, la negativa europea a la declaración unilateral de independencia por parte de Kosovo en 2008 y el envío de una misión a Kosovo para reformar el sistema judicial y la aplicación de la ley, el apoyo a Tiblisi tras la guerra entre Rusia y Georgia o el rechazo a la independencia de Osetia del Sur y Abjasia.

Hay que resaltar que, si bien en las cordiales relaciones entre la Unión y la Federación Rusa tras el fin de la Guerra Fría tuvieron mucho que ver los Estados de Europa Oriental, ahora nuevos miembros de la UE, otro tanto ha ocurrido posteriormente, cuando la relación con Rusia ha

La implicación de la UE en la región se ha mantenido firme incluso cuando abiertamente contradecía las preferencias de Rusia 
sido mucho más tirante. En efecto, en particular Estados como Polonia o las Repúblicas Bálticas no han dudado en apostar por una política de firmeza frente a Rusia, ya que consideran que tanto dicha firmeza como la vinculación del vecindario europeo a las estructuras de seguridad occidentales son elementos claves del mantenimiento de la propia seguridad europea (Merlingen, 2012, p. 225).

\section{Conclusiones}

Aun cuando pudiera parecer paradójico en un primer momento, el contexto estratégico que se abre al fin de la Guerra Fría supone un impulso a la cooperación en materia de seguridad y defensa en el seno de la Unión Europea. Sin embargo, ese impulso se verá en buena medida limitado como consecuencia de dos factores: la incorporación de nuevos miembros procedentes del antiguo "bloque oriental" a la Unión, con sensibilidades y culturas estratégicas muy diferentes, y la necesidad de posicionarse y actuar en los nuevos conflictos que surgen en la periferia europea.

Además, en los años transcurridos desde el fin de la Guerra Fría, el contexto estratégico no ha hecho sino complicarse, surgiendo fuentes de incertidumbre tanto desde el sur como del este de la Unión Europea.

Un desafío al que se une e creciente escepticismo con que algunos de los nuevos miembros (al menos las élites) han gestionado su participación en la UE, que ha supuesto indudablemente un obstáculo para el proceso de integración. Ese escepticismo, si cabe mayor en el ámbito de la seguridad y la defensa, también ha dificultado la cooperación en esta materia. Con todo parece claro que, en el nuevo contexto estratégico, a la vista de los crecientes desafíos a la seguridad a que la Europa debe hacer frente desde el sur, pero también desde el este y habida cuenta, además, de las posiciones de la Administración Trump y de la incertidumbre que se deriva de ellas acerca de lo que efectivamente cabe esperar de Washington, los nuevos miembros tendrían que revisar su enfoque.

Solo así sería posible avanzar hacia la integración también en lo que tiene que ver con la Política Exterior y de Seguridad Común y, muy en especial, la Política Común de Seguridad y Defensa, reduciendo la tendencia a la actuación autónoma de los Estados miembros y al recurso a mecanismos de flexibilidad que ahondan en la Europa a varias velocidades, como la PESCO, y debilitan el proceso de integración.

Por otra parte $-\mathrm{y}$ pese a los éxitos parciales que haya podido tener la Política de Vecindad primero y la Asociación Oriental después de la Unión Europea y a lo loable de la firmeza con la que, pese a consecuencias desagradables, ha actuado en algunos casos en el marco de dichas políticas - en muchos casos los resultados obtenidos con ellas han seguido lejos de lo deseable y de las propias aspiraciones de la UE.

Con todo, es innegable que la Unión Europea ha hecho de la necesidad virtud y desde el fin de la Guerra Fría, en un contexto que, pese a las apariencias, ha sido más adverso que nunca, ha dado un impulso a su cooperación en materia de seguridad y defensa. Ahora bien, la UE deberá no solo sostener ese impulso, sino lograr una mayor implicación de todos los miembros en él, incluidos los del sudeste europeo, pues no en vano cuentan con un peso presupuestario y demográfico nada desdeñable. No solo la integración en materia de seguridad y defensa, sino el avance del proceso de construcción europea en su conjunto, dependen de ello. 


\section{Referencias}

Arteaga, F. (2016) La defensa europea entre la Estrategia Global y su implementación, documento de trabajo 16/2016, Real Instituto Elcano, 28 de diciembre de 2016. http://www. realinstitutoelcano.org/wps/wcm/connect/381 1931c-83d8-42e5-a174-9a92c7c6aa51/ DT16-2016-Arteaga-Defensa-europea-Estrategia-global-implementacion.pdf?MOD=A JPERES\&cacheid=1482916405398

Bindi, F. (2012). European Union Foreign Policy: A Historical Overview. En F. Bindi y I. Angelescu, The Foreign Policy of the European Union. Washington: Brookings Institution.

Carrapiço, H. (2003-2004). The European Union's Eastern Enlargement: a Guarantee of Security or a Security Trap? Federalisme Regionalisme, 4. https://popups.uliege. be:443/1374-3864/index.php?id=220

Conclusiones de la Presidencia, Consejo Europeo de Helsinki 10 y 11 de diciembre de 1999, Recuperado de Internet (http://www.europarl.europa.eu/summits/hel1_es.htm)

Conclusiones de la Presidencia, Consejo Europeo de Laeken, 15 y 16 de diciembre de 2001, Recuperado de Internet (https://www.consilium.europa.eu/media/20950/68827.pdf)

Conclusiones de la Presidencia, Consejo Europeo de Madrid, 15 y 16 de diciembre de 1995, Recuperado de Internet (http://www.europarl.europa.eu/summits/mad3_es.htm

Conclusiones de la Presidencia, Consejo Europeo de Santa María de Feira, 19 y 20 de junio de 2000, Recuperado de internet (http://www.europarl.europa.eu/summits/fei2_es.htm)

Coricelli, F., Campos, N., y Moretti, L. (2015). European integration: Benefits of deep versus shallow integration. VOW-EU CEPR. https://voxeu.org/article/european-integrationbenefits-deep-versus-shallow-integration

Cornish, P., y Edwards, G. (2001). Beyond the EU/NATO dichotomy: the beginnings of a European Strategic Culture. International Affairs, 77(3), 587-603. https://doi. org/10.1111/1468-2346.00208

Criterios de Adhesión o Criterios de Copenhague. https://eur-lex.europa.eu/summary/glossary/ accession_criteria_copenhague.html?locale=es

Declaración del Consejo Europeo sobre el Refuerzo de la Política Europea Común de Seguridad y Defensa, Concusiones de la Presidencia, Consejo Europeo de Colonia, 3 y 4 de junio de 1999. (http://www.europarl.europa.eu/summits/kol2_es.htm\#bilIII)

Estrategia de Seguridad Europea, "Una Europa segura en un mundo mejor", Consejo de la Unión Europea, diciembre de 2003.

Estrategia Global Para la Política Exterior y de Seguridad de la Unión Europea. (2016). Una visión común, una actuación conjunta: una Europa más fuerte. Consejo de la Unión Europea.

Duchene, F. (1973). The European Community and the Uncertainties of Interdependence. En K. Max y H. A. Wolfgang, Nation Writ Large?Foreign Policy Problems before the European Community. Londres: McMillan. 
Fiott, D. (2018). Strategic autonomy: towards 'European sovereignty' in defence? EU Institute for Security Studies, Issue Brief 12/2018, París. https://www.iss.europa.eu/sites/default/ files/EUISSFiles/Brief\%2012Strategic\%20Autonomy.pdf

Fiott, D. (2017). European Security and Defence. Paris: The Basics, EU Institute for Security Studies. https://www.iss.europa.eu/content/european-security-and-defence$\%$ E2 \%80\%93-basics

Franco-British St. Malo Declaration, Joint Declaration on European Defence, 4 de diciembre de 1998. https://www.cvce.eu/obj/franco_british_st_malo_declaration_4_december_1998en-f3cd16fb-fc37-4d52-936f-c8e9bc80f24f.html

Forner, S. (2007). La Construcción de Europa: De las "guerras civiles" a la unificación. Madrid: Biblioteca Nueva

Gati, C. (2007). Backsliding in Central and Eastern Europe. Connections, 6(3), 107-120. https://doi.org/10.11610/connections.06.3.07

Gaub, F. (ed.). (25 January 2019), What if...? Scanning the horizon: 12 scenarios for 2021, European Union Paris: Institute for Security Studies. https://www.iss.europa.eu/content/ what-if-scanning-horizon-12-scenarios-2021

Garrido V. (2010) Evolución histórica de la seguridad y la defensa europea de la PESC a la PCSD. En CESEDÉN, El futuro de las Fuerzas Multinacionales Europeas en el marco de la nueva politica de Seguridad y Defensa. Madrid: Ministerio de Defensa.

Garrido, V. (2015). Balance de la Política de Seguridad y Defensa de la UE después del Tratado de Lisboa: Principales aportaciones del Tratado de Lisboa al desarrollo de la PCSD. En F. Aldecoa y J. M. Gil Robles (coords.), Profundicemos sobre Europa, VI Curso de Verano en Pozuelo (pp. 67-79). Madrid: UCM-Centro de Excelencia Jean Monnet "Antonio Truyol".

Garrido, V. (2019). La política exterior y de seguridad común (PESC). En C. Fernández de Casadevante, F. J. Carrera Hernández, J. Ruiloba Alvariño, y V. Garrido (coords.). Derecho de la Unión Europea. Nociones Básicas de la Unión Europea. Madrid: Editorial Universaria Ramón Areces.

Hopi, O. (2011/2012). The EU Foreign and Security Policy after the Cold War. Search of Identity. POLIS Journal, 6.

Ischinger, W. (25 de junio 2009). The European Security Architecture two decades after the fall of the Berlin Wall: can Russia be integrated? SIPRI. https:/www.sipri.org/commentary/ essay/thu-06-25-2009-14-00/european-security-architecture-two-decades-after-fall-ofberlin-wall-can-russia-be-integrated

Jones B. (2020). "CSDP defence capabilities development" In Depth AnalysesEuropean Parliament January. https://www.europarl.europa.eu/RegData/etudes/ IDAN/2020/603482/EXPO_IDA(2020)603482_EN.pdf

Kaldor, M. (1999). Old and New Wars: Organized Violence in a Global Era. Cambridge: Polity Press.

Keukeleire, S., y Macnaughtan, J. (2008). The Foreign Policy of the European Union. Basingstoke: Palgrave McMillan. 
Kocamaz, S. (2011). A new form of security cooperation and collective conflict management in the Post Cold War International System. International Journal of Social Sciences and Humanity Studies, 3(1), 467-476.

Kolankiewicz, G. (1994). Consensus and Competition in the Eastern Enlargement of the European Union. International Affairs, 70(3), 477-495. https://doi.org/10.2307/2623707

Mangas Martín, A. (1989). La Unión Europea Occidental. Anuario CIDOB, 75-79

Mazziotti, M. (2014). The European Security Architecture 1990-2014: Old Approaches to New Problems? CBEES Annual Conference.

Merlingen, M. (2012). EU Security Policy. Boulder: Lynne Rienner.

Muti, K. (2018, septiembre). Poland: The Missing Link in European Defence. IAI Commentaries, $18(48)$.

Park, J. (13 de marzo 2014). The European Union's Eastern Partnership. Council of Foreign Relations. https://www.cfr.org/backgrounder/european-unions-eastern-partnership

Petersberg Declaration, Western European Union, Council of Ministers, Bonn, 19 june 1992. http:/www.weu.int/documents/920619peten.pdf

Rodríguez Prieto, V. (2018). La actuación normativa de la Unión Europea en la Asociación Oriental: Los casos de Georgia y Moldavia (2009-2018). Madrid: Marcial Pons

De Castro Ruano, J. L. (2020). La Integración de la seguridad y la defensa en la unión europea. Instituto Robert Schumann de estudios europeos. Madrid: Universidad Francisco de Vitoria.

Ruiz González, F. J. (2010). "Las Relaciones Unión Europea-Rusia, la "Asociación Oriental”, el futuro de la OSCE, y sus consecuencias para la Política Común de Seguridad y Defensa (PCSD). En VV. AA., La Política Europea de Seguridad y Defensa (PESD) tras la entrada en vigor del Tratado de Lisboa, Cuadernos de Estrategia 145, Instituto Español de Estudios Estratégicos. Madrid: Ministerio de Defensa

Sánchez, V. M. (dir.). (2010). Derecho de la Unión Europea. Barcelona: Huygens

Saulnier, F., Anghel, S., Lazarou, H., y Wilson, A. W. (2020). On the path to 'strategie autonomy': The EU in an evolving geopolitical environment. European Parliament Report on Security (EPRS), https://www.europarl.europa.eu/RegData/etudes/STUD/2020/652096/EPRS_ STU(2020)652096_EN.pdf

Sjursen, H. (15 de diciembre de 1999). The Common Foreign and Security Policy: an Emerging New Voice in International Politics? ARENA Working Papers, WP 99/34. https://www. sv.uio.no/arena/english/research/publications/arena-working-papers/1994-2000/1999/ wp99_34.htm

Smith, J. (2009). How 1989 changed the history of European Integration. European View, 8, 255-262. https://doi.org/10.1007\%2Fs 12290-009-0096-4

Smith, K. E. (2004). European Union Foreign Policy in a Changing World. Cambridge: Polity Press.

Wallace, W. (2017). European foreign policy since the Cold War: how ambitious, how inhibited? The British Journal of Politics and International Relations, 19(1), 77-90. https://doi. org/10.1177\%2F1369148116685297 
Wallace, W. (2000). From the Atlantic to the Bug, from the Arctic to the Tigris? The Transformation of the EU and NATO. International Affairs, 76(3), 475-493. https://doi. org/10.1111/1468-2346.00147

Wienner, A., y Diez, T. (2004). European Integration Theory. Oxford: Oxford University Press. 